\title{
Wound Closure on the Neonatal Rat Skin II. The Potential Ability of Epidermis to Close Small-Sized Wounds Independently of the Underlying Dermis
}

\author{
Mary Arai, Takashi Matsuzaki, Setsunosuke Ihara* \\ Department of Biological Science, Faculty of Life and Environmental Science, Shimane University, Matsue, Japan \\ Email: ${ }^{*}$ ihara@life.shimane-u.ac.jp
}

Received September 24, 2013; revised October 24, 2013; accepted November 1, 2013

Copyright (C) 2013 Mary Arai et al. This is an open access article distributed under the Creative Commons Attribution License, which permits unrestricted use, distribution, and reproduction in any medium, provided the original work is properly cited.

\begin{abstract}
Reepithelialization of skin which comprises epidermis and dermis has not been fully elucidated due to the complexity of the participants as well as the interactions therein. In this study, the intrinsic roles and behaviors of epidermis itself during wound closure on neonatal rat skin were explored by developing and utilizing a novel in vivo wound model, termed "shallow incisional wound" in which the injury of dermis was minimized. The shallow wounds were closed by $12 \mathrm{~h}$ postwounding (PW) by the migration of the wound-marginal epidermal sheets in which activated myosin light chain (p-MLC) was predominantly detected at the lateral plasma membrane of individual cells. By local administration of Rho-associated protein kinase (ROCK) inhibitor Y27632, p-MLC disappeared at the wound margin and wounds were not closed by $12 \mathrm{~h} \mathrm{PW}$. Inhibition of Rac 1 by NSC23766 also resulted in hold of wound closure by $12 \mathrm{~h} \mathrm{PW}$, though NSC23766 somewhat slowly acted on p-MLC expression. These results suggest that, without joining of dermis, epidermal cells have a potential ability of closing wounds by active epithelial sheet movement integrated by Rho family small GTPases-dependent extension and contraction of the individual cell bodies.
\end{abstract}

Keywords: Wound Closure; Reepithelialization; Rat; Neonate; Epidermis; Migration; Actomyosin; Rho Family Small GTPases

\section{Introduction}

Skin is located at the outermost layer of multicellular organisms, and plays a definite role in the maintenance of homeostasis [1,2]. When skin is injured, the rapid repair is needed to prevent invasion and water loss. Wounds on adult mammalian skin are closed by epidermal cells that crawl from the wound edges [3].

A series of our previous study showed that full-thickness excisional wounds made on neonatal rats were covered with quick reepithelialization by the marginal epidermis, and that immunohistochemical analyses using anti-keratin and cadherin antibodies could discriminate the major cell populations of the epidermal cells covering the wounds into the basal cell-derived "leading edge cells" and "immature spinous cells" [4,5]. Most recently, we showed that, during the full thickness incisional wound healing on neonatal rats, the thickness of the epidermis was fluctuated (regulated) at least by the delay

*Corresponding author. and acceleration of the terminal differentiation, including cornification, of the epidermal keratinocytes [6]. However, many parts of the mechanisms involved in the reepithelialization process still remain to be elucidated, because this kind of study encounters many difficulties due to the complexity of the experimental system, such as multiplicity of participants therein and the influences of type and geometry of wounds, and so on.

In this study, we first concentrated on making the incisional wounds as simple as possible so that we could pursue the essential behavior of epidermis in the epithelial migration. Through try and error, we established a wounding design, termed here "shallow incisional wound", by which only the epidermis and a upper-third of dermis were incised, keeping the lower part of skin intact. The shallow incisional wounds made on neonatal rat skin were immunohistochemically analyzed by use of antibodies of actin-myosin cytoskeleton, cadherins, and keratin.

It is known that the regulation of actin cytoskeleton by 
Rho small GTPases is indispensable for epithelial migration [7-9]. In migrating cells, activated Rac facilitates lamellipodia formation, and cells extend forward at the front of cells, whereas activated Rho forms stress fibers, focal adhesions, and cells contract by phosphorylation of myosin at the backward [10]. The balance between activities of these two Rho small GTPases is considered to be critical for migration and formation of cells, and the actions of Rac 1 and Rho A antagonize and downregulate each other [10,11-13]. However, these findings were largely based on analyses using culture systems in vitro, and validation in vivo is poorly made except for only a few instances $[10,14]$. Therefore, we examined the impact on wound closure when the pathways of Rho A or Rac 1 were locally inhibited in the shallow incisional wounds. The experimental results obtained in this study strongly support the possibility that wound closure could be executed by the active migration of the only epidermis, independently of dermis, on neonatal rat skin.

\section{Materials and Methods}

\subsection{Wounding}

Sprague-Dawley rats were anesthetized at 1 day after birth. Two incisional wounds were made on the back skin on both sides of the dorsal midline with glaucoma scalpels, MICRO FEATHER No. 6320G (FEATHER). The wound sites were immediately covered with the cover agent Opsite (Smith \& Nephew), and the wounded individuals were returned to the mother rats. The Opsite treatment had no effects on the wound healing except that it avoided unnecessary retardation of healing due to the infection that otherwise occasionally occurred [4].

Adult rats were inflicted with several wounds in a similar way, though the Opsite was not applied.

\subsection{Histology and Immunohistochemistry}

The skin surrounding the wound was cut out at $0,6,8,10$, 12 , or $18 \mathrm{~h} \mathrm{PW}$. Pieces of the skin were fixed with $4 \%$ paraformaldehyde in $\mathrm{Ca}^{2+}, \mathrm{Mg}^{2+}$-free phosphate-buffered saline $[\mathrm{PBS}(-)]$ for $1 \mathrm{~h}$ at room temperature, and embedded in paraffin. Sections ( $4 \mu \mathrm{m}$ thickness) were subjected to Masson's trichrome (MT) staining or immunohistochemical analyses. For immunohistochemistry, deparaffinized sections were pretreated with citric acid buffer (pH 6.0) or $1 \mathrm{mM}$ EDTA ( $\mathrm{pH} 8.0$ ) at $95^{\circ} \mathrm{C}$, and rinsed with $\mathrm{PBS}(-)$. Alternatively, the sections were pretreated with $2 \mathrm{~N} \mathrm{HCl}$, followed by digestion with $0.05 \%$ trypsin at $37^{\circ} \mathrm{C}$. The pretreated sections were incubated with $1 \%$ normal horse serum in PBS(-) for $20 \mathrm{~min}$, and then reacted with primary antibodies at $4^{\circ} \mathrm{C}$ overnight. They were washed with $\operatorname{PBS}(-)$, then incubated with secondary antibodies for $2 \mathrm{~h}$. Samples were washed with
PBS(-), counterstained with 4',6-diamino-2-phenylindole dihydrochloride (DAPI, Polysciences) at $0.1 \mu \mathrm{g} / \mathrm{ml}$, and mounted. The following primary antibodies were used: mouse anti-cytokeratin 6 monoclonal antibody $(\mathrm{mAb})$ (Progen, 1:50); mouse anti-E-cadherin mAb (BD Transduction Laboratories, 1:500); goat anti-mouse P-cadherin polyclonal antibody (pAb) (R \& D Systems, 1:100); rabbit anti-human myosin light chain (phosphor S20) pAb (abcam, 1:500); rabbit anti-myosin pAb (SIGMA, 1:100); and mouse anti-actin mAb (MILLIPORE, 1:100). Alexa fluor 594 goat anti-mouse $\operatorname{IgG}_{1}$ (Molecular Probes, 1:1500); Alexa fluor 488 goat anti-mouse $\operatorname{IgG}_{2 \mathrm{a}}$ (Molecular Probes, 1:500); Alexa fluor 594 donkey anti-goat IgG (Molecular Probes, 1:1500); and Alexa fluor 488 goat anti-rabbit IgG (Molecular Probes, 1:500) were used as secondary antibodies.

The staining of fibrinogen was performed using immunoenzyme technique with rabbit anti-fibrinogen (ABIN, 1:500) and HRP-conjugated anti-rabbit immunogrobulins (DakoCytomation, 1:100).

Lectin staining for visualizing the intercellular borders in the rat epidermis $[15,16]$ was performed with biotinylated peanut agglutinin (PNA) (VECTOR LABORATORIES, 1:200) and Texas Red conjugated streptavidin (Thermo SCIENTIFIC, 1:1000).

\subsection{Administration of ROCK and Rac 1 Inhibitors}

ROCK inhibitor Y27632 (SIGMA, dissolved in water) and Rac 1 inhibitor NSC23766 (Calbiochem, dissolved in dimethyl sulfoxide) were dissolved at final concentrations of $0.7 \mathrm{mM}$ and $0.5 \mathrm{mM}$, respectively, in $30 \%$ Pluronic F-127 (Invitrogen). Pluronic F-127 at this concentration is known to be gelated at body temperature [17, 18]. The chilled mixtures of Pluronic F-127 and the inhibitors were locally applied to the neonatal skin wounds at the dose of $10 \mu \mathrm{l}$ per wound immediately after wounding. The wounds were covered with the cover agent Opsite, and the wounded individuals were returned to the mother rats. The control experiments were also done using water or dimethyl sulfoxide instead of the inhibitor solutions.

\section{Results}

\subsection{The Morphology of the Shallow Incisional Wounds Healing on Neonatal Rat Skin}

MT staining indicated that the shallow incisional wounds made by the present technique had been cut from epidermis to papillary dermis, and that the lower part of dermis and panniculus carnosus had remained intact (Figure 1(A)). From $6 \mathrm{~h}$ PW onward, the wound edges of epidermis became round, and the full-thickness epider- 


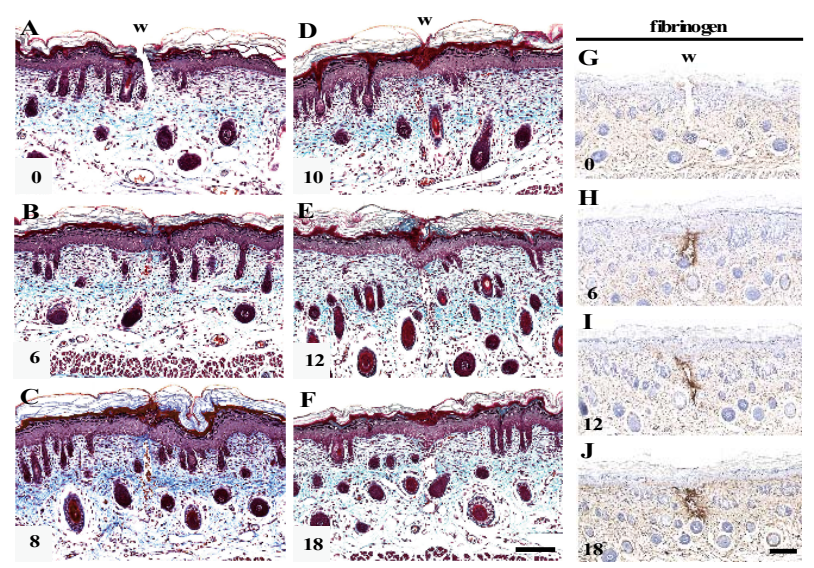

Figure 1. Morphological changes in the shallow incisional wounds on the neonatal rat skin during reepithelialization. One day-old neonatal skin was wounded by the technique for the shallow incisional wound described in Materials and Methods, and fixed at 6, 8, 10, 12, and $18 \mathrm{~h}$ PW. Cross sections were prepared and subjected to Masson's trichrome staining. In this technique, the incision reached the upper dermis, while the lower dermis and cutaneous muscle remained uninjured, as seen in the $0 \mathrm{~h} P W$ wound (A). At $6 \mathrm{~h}$ $P W$, the epidermal wound edges were rounded and approached each other $(B)$. The leading edges of epidermis were more migrated toward the wound center or mutually contacted during the period from about 8 to $10 \mathrm{~h} \mathrm{PW}$, as seen in (C) (8 h PW) and (D) (10 h PW). Around $12 \mathrm{~h} \mathrm{PW,}$ the epidermal wound closure was completed $(\mathrm{E})$, whereas gaps frequently remained just below dermis $((E),(F))$. These dermal gaps were immunohistochemically positive for fibrin from $6 \mathrm{~h}$ PW onward ((G)-(J)). Scale bars, $100 \mu \mathrm{m}$.

mis was gradually migrating toward the center of wound, and these migrating tongues contacted each other (Figures 1(B)-(D)). The wound closure by reepithelialization was completed by $12 \mathrm{~h}$ PW (Figure 1(E)).

At the dermis below the wounds, small gaps were left in many sections from $6 \mathrm{~h}$ PW onward, even after wound closure by epidermis (Figures 1(B)-(F)). In these gaps, erythrocytes and/or nucleated cells were occasionally observed, but collagen fibers stained with aniline blue were absent. The immunohistochemical analyses suggested the presence of fibrin in the dermal gaps from $6 \mathrm{~h}$ PW onward (Figures 1(G)-(J)).

\subsection{The Expression of Cadherins and Keratin}

In the wounds just after operation, E- and P-cadherins were localized from the basal to the granular layers and the only basal layer, respectively, in the same manner as in the normal skin (Figures 2(A) and (G)). The expression zone of E-cadherin remained unchanged from $6 \mathrm{~h}$ PW onward (Figures 2(B)-(F)). On the other hand, the expression zone of P-cadherin was enlarged by 2 - 4 cell layers apical at $6 \mathrm{~h} \mathrm{PW}$ (Figure 2(H)). At $10 \mathrm{~h} \mathrm{PW}$, the enlargement pattern of P-cadherin expression up to the
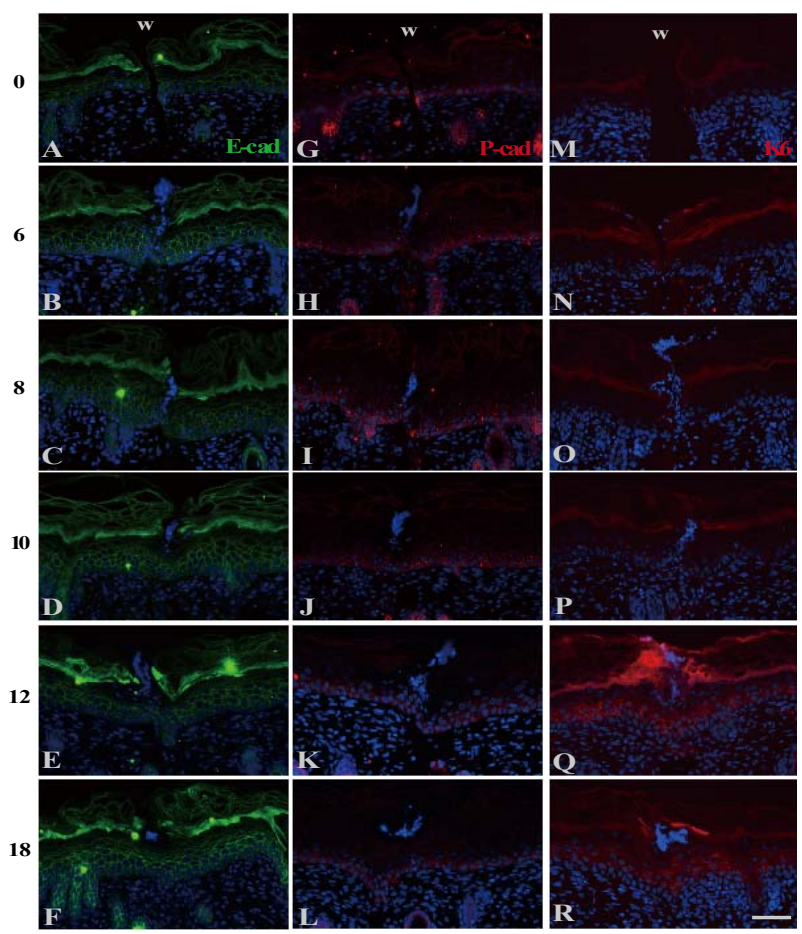

Figure 2. The localization of cadherins and keratins in the wound areas of the neonatal epidermis. E-((A)-(F)) and Pcadherin $((G)-(L))$ and keratin $6((M)-(R))$ were immunohistochemically detected. Just after wounding, E-cadherin and $P$-cadherin were localized at the basal and suprabasal layer (A), and the basal layer only (G), respectively. K6 keratin was undetectable at $0 \mathrm{~h}$ PW (M). The normal patterns of E-cadherin (B) and K6 (N) lasted until 6 h PW. On the contrary, P-cadherin-positive zone were expanded upward by 2 to 4 cell layers till then $(H)$. At 10 h PW, P-cadherin-dilation region was restricted within $400 \mu \mathrm{m}$ in width $(\mathrm{J})$, while $\mathrm{K} 6$ began to express in the suprabasal layers at the region expanding from the wound edge outward $(P)$. By $12 \mathrm{~h}$ PW, the distribution of P-cadherin was further confined to the wound margin $(\mathrm{K})$, whereas that of $\mathrm{K6}$ was more broadened far from the wound areas $(Q)$. Scale bar, $50 \mu \mathrm{m}$; counterstain, DAPI; and $W$, wound.

suprabasal layers was confined to within $400 \mu \mathrm{m}$ from wounds, while the normal pattern was observed in further distant regions (Figure 2(J)). From 12 to $18 \mathrm{~h} \mathrm{PW}$, the multilayered expression of P-cadherin was further restricted to the wound edges, and the wound-closed epidermis restored the normal single-layered expression pattern (Figures 2(K) and (L)).

At 0 and $6 \mathrm{~h}$ PW, K6 keratin was absent at the wounded epidermis as in the normal epidermis (Figures $\mathbf{2}(\mathbf{M})$ and $(\mathbf{N})$ ). K6 was detectable at the spinous layer of the wound margins in some 8 and $10 \mathrm{~h}$ PW sections (Figures 2(O) and (P)). At 12 and 18 h PW, K 6 expression was extended to not less than $400 \mu \mathrm{m}$ far from the wound margins (Figures 2(Q) and (R)). It was likely that, in the shallow incisional wounds, the initiation of $\mathrm{K} 6$ expression was delayed by several hours, as compared 
with the excisional wounds on neonates in which $\mathrm{K} 6$ was detectable from $6 \mathrm{~h}$ PW [4].

\subsection{Myosin Activation Was Involved in the Epidermal Sheet Migration during the Wound Closure}

It is generally known that cell migration is accomplished by a coordination of the following two intracellular events: 1) the formation of lamellipodia (and filopodia) through actin polymerization at the anterior side of the cell, and 2) the contraction of the cell body at the posterior side occurring via phosphorylation of myosin II $[10,19]$. Accordingly, we analyzed the expression of actin, myosin II and activated myosin light chain (p-MLC).

In the epidermis just after wounding, p-MLC was immunohistochemically undetected (Figures $\mathbf{3}(\mathbf{A})$ and $(\mathbf{F})$ ), even though actin and myosin II were enough stored (see Figures 4(A) and (E)). By 6 h PW, p-MLC became detectable predominantly at the lateral plasma membranes of the basal and the spinous cells in the range of 10 - 20 cells from the wound edges (Figures 3(B) and (G)). At that time, PNA lectin was costained to visualize plasma membranes. p-MLC was disclosed to be localized just beneath the lateral membranes of epidermal cells possibly moving toward wounds (Figure 3(K)). Subsequently ( 8 to $10 \mathrm{~h} \mathrm{PW}$ ), p-MLC became confined in the vicinity of wound edges, and almost disappeared around $12 \mathrm{~h} \mathrm{PW}$, when wound closure was completed (Figures 3(C)-(E) and (H)-(J)). On the other hand, the intracellular contents of actin and myosin II remained unchanged during wound closure (Figures 4(B)-(D) and (F)-(H)). The results from these analyses suggest that the myosin phosphorylation which actually drives the contraction of cells located at the lower part of epidermis may be a prerequisite for the centripetal epidermal migration, i.e., the closure of the shallow incisional wounds, on neonatal rat skin.

\subsection{The Difference between the Newborn and Adult Patterns of Incisional Wound Healing}

MT staining showed that the shallow incisional wounds in adults were dilated remarkably by $6 \mathrm{~h}$ PW, so that their wound closure was not finished until $18 \mathrm{~h}$ PW (Figures 5(A)-(D)). However, immunohistochemical analyses exhibited that, also in adult wounds, p-MLC appeared along the lateral of cell membranes of epidermal cells at 6, 12 and $18 \mathrm{~h} \mathrm{PW}$ (Figures 5(F)-(H)).

\subsection{ROCK Inhibition Induced Suppression of the Wound Closure}

In order to verify whether or not the closure of shallow incisional wounds relied on phosphorylation of myosin light chain (MLC), we further pursued a series of inhibi-
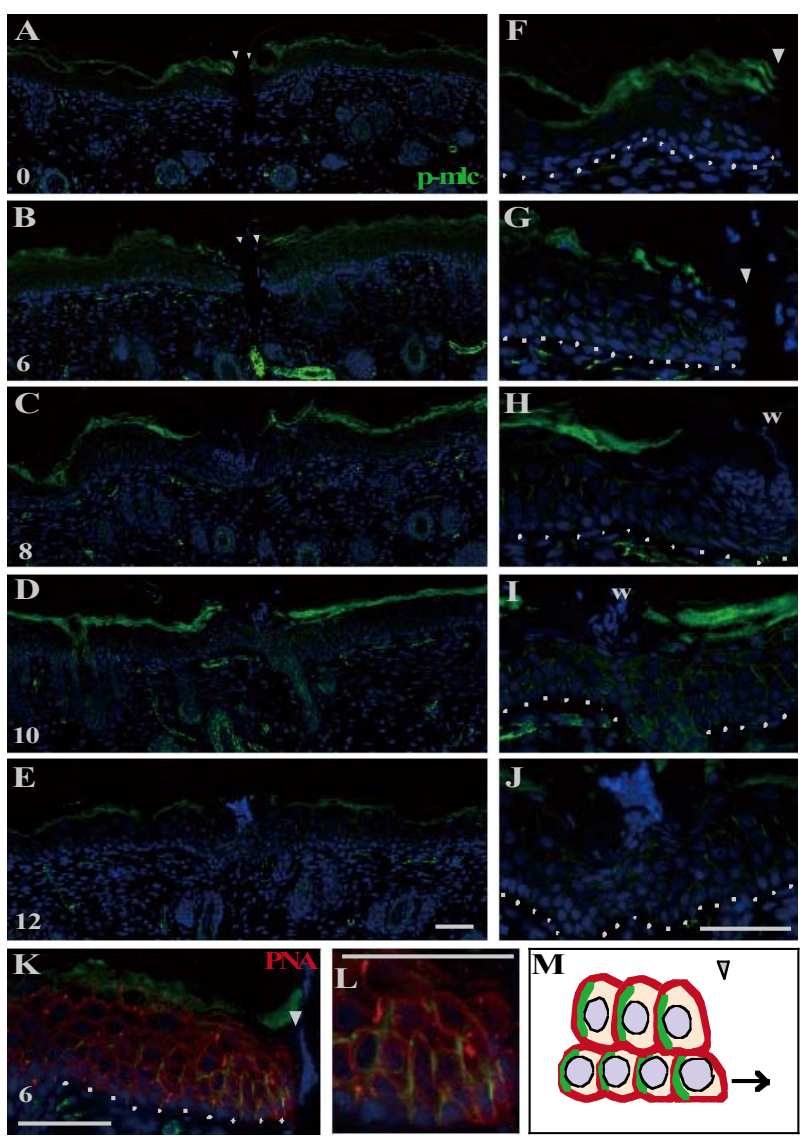

Figure 3. Myosin II was activated in the epidermal keratinocytes located at the wound region during the neonatal wound closure. The right panels $((F)-(L))$ are the magnifications of the wound margins shown in the left panels ((A)-(K)). p-MLC was undetectable at 0 h PW ((A), (F)). At $6 \mathrm{~h}$ PW, p-MLC became detectable at the lateral side of the basal and spinous cells around the wound margin $((B),(G))$. By 8 - $10 \mathrm{~h}$ PW, the expression of p-MLC was gradually confined to the wound edge ((C), (D), (H), (I)). From $12 \mathrm{~h}$ PW onward, p-MLC became almost absent in the epidermal cells $((\mathrm{E}),(\mathrm{J}))$. The double staining of $\mathrm{p}$-MLC and lectin PNA (peanut agglutinin) displayed that $p-M L C$ was localized in proximity to the lateral plasma membrane at the wound margin ((K), (L)). p-MLC probably also expressed at the posterior lateral side of cells in this system, as known in (M). Scale bars, $50 \mu \mathrm{m}$; counterstain, DAPI; arrowheads, wound edge; and dashed lines, the border between the epidermis and dermis.

tion experiments in which Y27632 was swabbed as inhibitor of ROCK to the wound-surrounding epithelium locally just after wounding.

Neither the Y27632-treated wounds nor the control wounds adhered their wound edges at $8 \mathrm{~h} \mathrm{PW}$ (Figures 6(A) and (F)). At that time, p-MLC was present at the epidermis of the wound margin in the control wounds (arrows in Figure 6(D)), but it was absent or very weakly positive in the Y27632-treated wounds (Figure 6(I)). At $12 \mathrm{~h} \mathrm{PW}$, wound closure by epidermis was finished in the 

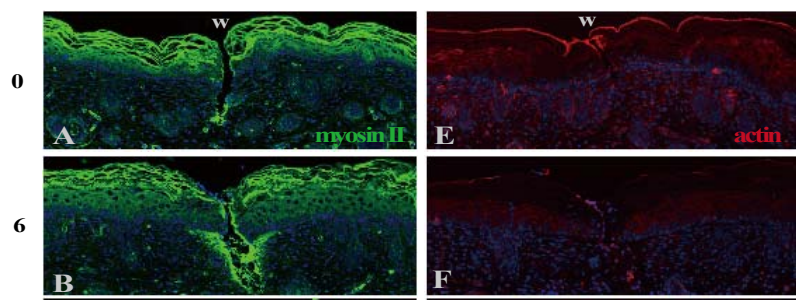

12
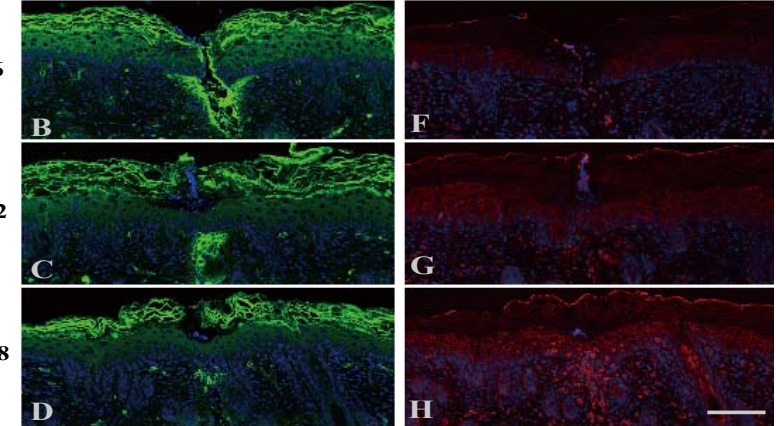

Figure 4. The expression levels of myosin II and actin were almost unchanged in the epidermis during the neonatal wound closure. Cross sections of neonatal skin were stained with anti-myosin II or anti-actin antibodies at the indicated post-wounding hours. Both non-muscle myosin II ((A)-(D)) and actin $((\mathrm{E})-(\mathrm{H}))$ were present at the epidermis in all the sections. Scale bar, $100 \mu \mathrm{m}$; and counterstain, DAPI.
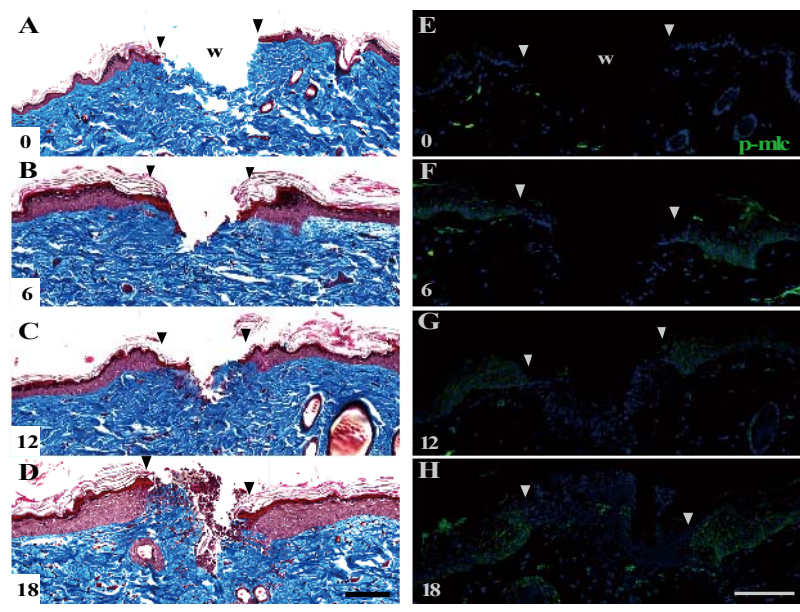

Figure 5. The shallow incisional wounds on the adult rat skin. Cross sections of the adult skin inflicted with the shallow incisional wounds were subjected to Masson's trichrome staining ((A)-(D)) and anti-p-MLC staining (E-H). The wounds were not completed even at $18 \mathrm{~h}$ PW (D). Meanwhile, p-MLC was distributed in the wound areas of epidermis during the period from 6 to $18 \mathrm{~h} \mathrm{PW}((\mathrm{E})-(\mathrm{H}))$. Scale bars, $100 \mu \mathrm{m}$; counterstain, DAPI; and arrowheads, wound edge.

control wounds, while the epidermal wound edges remained detached in the Y27632 treated wounds (Figures 6(B) and (G)).

p-MLC at the epidermal wound margin in the control wounds became almost undetectable (Figure 6(E); see also Figures 3(E) and (J)). On the contrary, the Y27632treated wounds had a tendency to be beginning to express $\mathrm{p}$-MLC in the epidermal wound margin, though infrequently (arrows in Figure 6(J)). By 18 h PW, wound

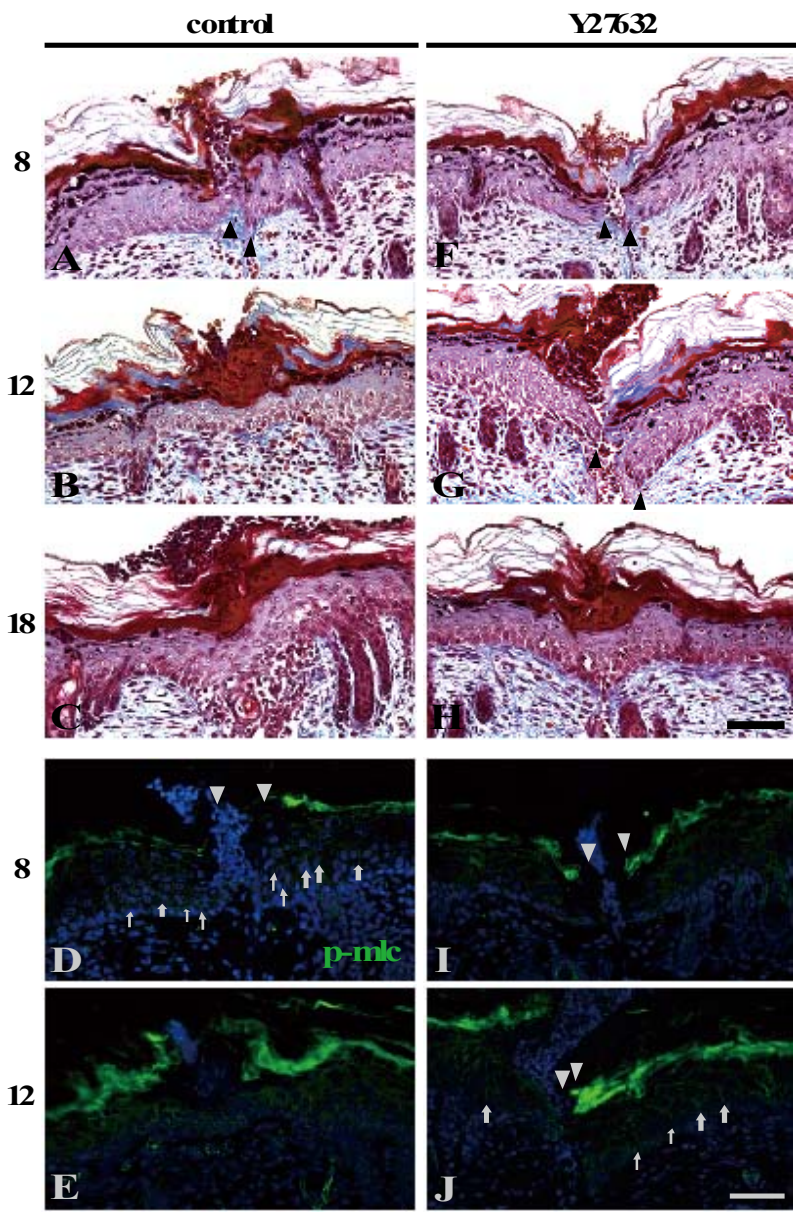

Figure 6. Phosphorylation of myosin light chain was required for the closure of the shallow incisional wounds. The ROCK inhibitor Y27632-administered ((F)-(J)) or unadministered ((A)-(E)) shallow incisional wounds in the neonatal skin at 8, 12, and $18 \mathrm{~h}$ PW were subjected to Masson's trichrome staining and anti-p-MLC staining. Both of the Y27632-treated and the control wounds were not closed at 8 h PW ((A), (F)). On the other hand, p-MLC was detected well in the control wounds ((D), arrows), but it was absent or very weakly detected, if any, in the Y27632-treated wounds (I). The control wounds were closed by 12 h PW (B) and the p-MLC expression in the epidermis disappeared $(\mathrm{E})$. It should be noted that the Y27632-treated wounds at 12 h PW were still opened (G), but apt to become p-MLC-positive at the wound edges, though not frequently ((J), arrows). The wound closure was completed in the Y27632-treated wounds by 18 h PW (H). Scale bars, $50 \mu \mathrm{m}$; counterstain, DAPI; arrows, p-MLC; and arrowheads, wound edge.

closure was completed even in the presence of Y27632 (Figure 6(H)). It was thought that p-MLC expression and closure in the Y27632-treated wounds from $12 \mathrm{~h} \mathrm{PW}$ onward were caused by the reversibility and instability of the inhibitor. Through all the time points, the staining patterns of actin and E-cadherin were largely unchanged (data not shown). These results from the inhibition experiments matched the above-stated reasoning (from the 
results shown in Figure 3) that the directional contraction of cells by myosin may be required for the wound closure in the shallow incisional wounds.

\subsection{The Inhibition of Rac 1 Prevented the Wound Closure}

Lastly, we examined the influences of the activity of Rac 1 on the incisional wound closure. Rac 1 is known to be activated at the front of migrating cells, participate in cellular extension, and further antagonize with Rho A. NSC23766, an inhibitor of Rac 1, was applied locally to the wounded skin just after wounding in the same way as in the previous experiments with Y27632.

Neither the NSC23766-treated wounds nor the control wounds adhered their wound edges at $8 \mathrm{~h}$ PW (Figures 7(A) and (F)). Unlike the Y27632 treatment, both the NSC23766-treated and the control wounds were positive for $\mathrm{p}$-MLC at the wound edges of epidermis (arrows in Figures 7(D) and (I)). By $12 \mathrm{~h} \mathrm{PW}$, the control wounds finished wound closure and lost the amount of p-MLC (Figures 7(B) and (E)). Meanwhile, the NSC23766treated wounds were still open, though poor in p-MLC like the control wounds (Figures $7(\mathbf{G})$ and $(\mathbf{J})$ ). Wound closure was completed in the NSC23766-treated wounds by $18 \mathrm{~h}$ PW (Figure $\mathbf{7 ( H )})$. There was no difference in the strength of actin staining between the NSC23766treated and the control wounds at any time point (data not shown). These results suggested that, although, unlike Y27632, NSC23766 did not quickly prevent the expression of $\mathrm{p}-\mathrm{MLC}$ under the present administration conditions, the administration of this Rac 1 inhibitor also resulted in a delay of the shallow incisional wound closure on rat neonates.

\section{Discussion}

\subsection{General View of the Macroscopic and Morphological Features of Wound Closure in the Shallow Incisional Wounds on Neonatal Rats Skin}

In this study, the closure of the shallow incisional wounds on neonatal rats' skin was completed by $12 \mathrm{~h} \mathrm{PW}$ (Figures 1 and $\mathbf{8 ( A )}$ ). It was a rapid response in comparison to that of the shallow incisional wounds made by the same operation techniques on adult rats which did not close at least $18 \mathrm{~h}$ PW (Figure 5) or that of the fullthickness incisional wounds on the neonates which was closed by 48 h PW [6]. Additionally, there were no signs of the crawling of epidermal cells on the wound bed, which is generally regarded as an attribute of adult wounds. Such a morphological feature of the shallow incisional wounds was thought to be due to the fact that the injury to dermis was minimum so that the crawling of

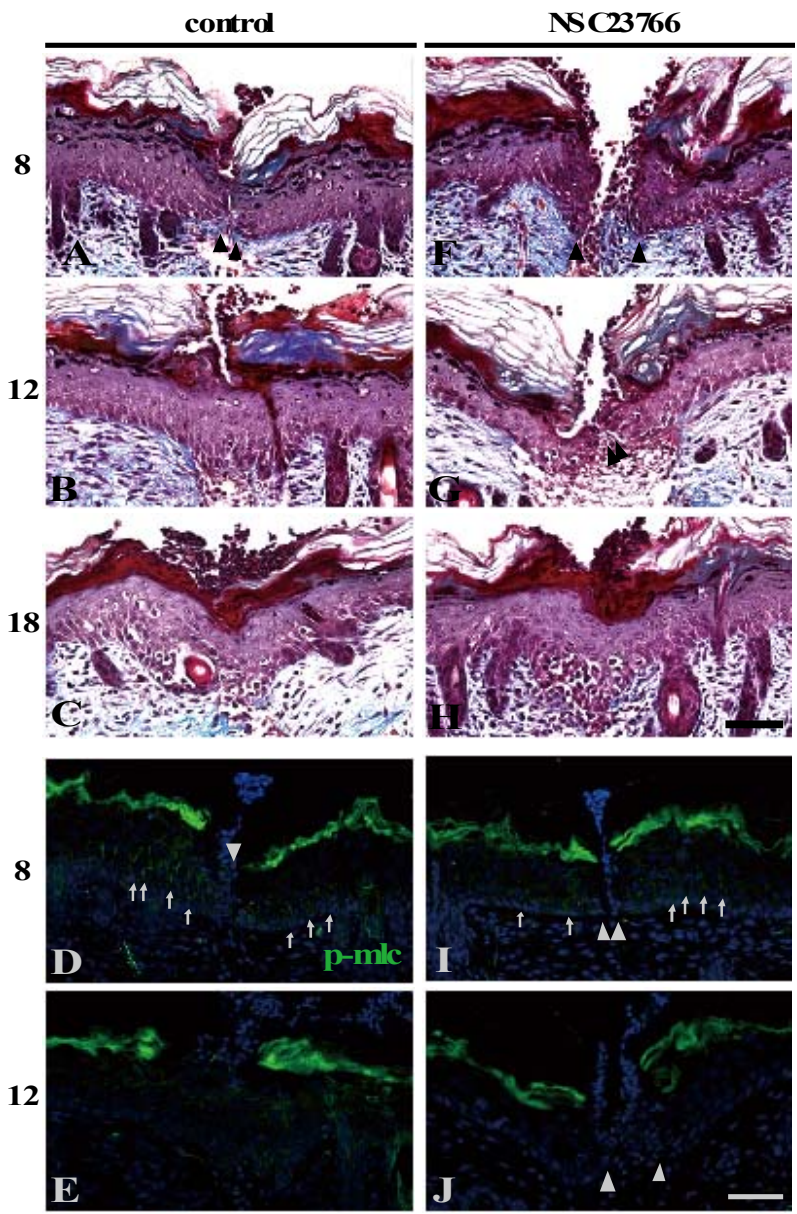

Figure 7. The wound closure was prevented by Rac 1 inhibition. The Rac 1 inhibitor NSC23766-administered ((F)-(J)) or unadministered ((A)-(E)) shallow incisional wounds in the neonatal skin at 8,12 , and $18 \mathrm{~h} \mathrm{PW}$ were subjected to Masson's trichrome staining and anti-p-MLC staining. By 8 $h$ PW, both of the NSC23766-treated and the control wounds were not only still open $((\mathrm{A}),(\mathrm{F}))$, but also p-MLCdetectable to a similar extend ((D), (I)), unlike the Y27632 treatment (see Figures 6(D), (I)). At 12 h PW, the control wounds were closed $(B)$ and the p-MLC expression in the epidermis disappeared (E), whereas the NSC23766-treated wounds at that time were still opened (G), though p-MLC was already absent $(J)$, like the control wounds. The wound closure was completed in the NSC23766-treated wounds by $18 \mathrm{~h}$ PW (H). Scale bars, $50 \mu \mathrm{m}$; counterstain, DAPI; arrows, p-MLC; and arrowheads, wound edge.

epidermal cells was unwanted for covering the gap between the wound edges.

\subsection{Wound Closure Depends on the Activation of Actomyosin System in the Epidermal Cells at Wound Edges}

The active form of myosin (p-MLC) was observed beneath the lateral plasma membranes in the migrating epidermis around the wound edges, and disappeared after 


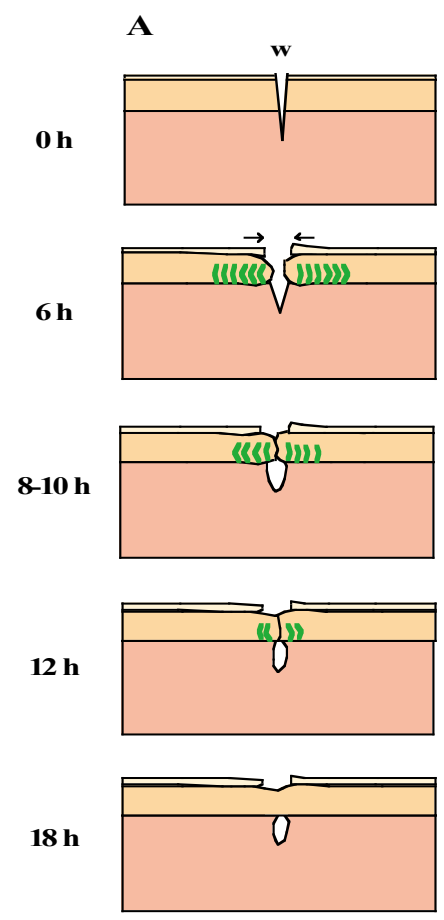

B
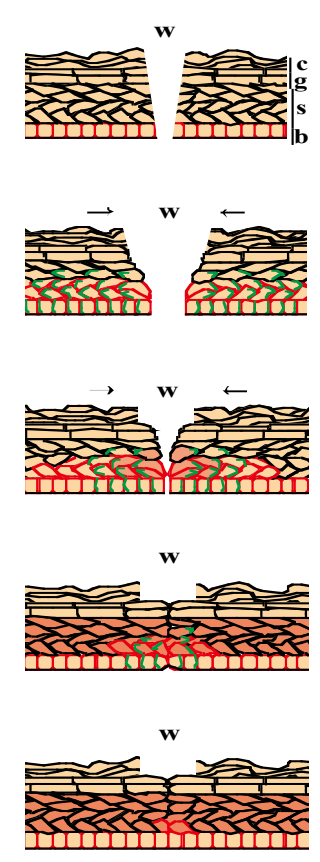

Figure 8. The scheme of wound closure in the shallow incisional wounds on the neonatal rat skin. The results from the morphological and immunohistochemical analyses are illustrated (A, epidermis and dermis; B, epidermis). The epidermal stratification at $\mathrm{O} \mathrm{h} \mathrm{PW}$ is arrayed in the same order as that in the normal skin. By $6 \mathrm{~h}$ PW, the epidermis of the wound edge starts to migrate toward the wound center, p-MLC becomes detectable at the lateral side of the basal and spinous cells, and the expression of P-cadherin is expanded up to the suprabasal layer around the wound region. Thereafter (8 to $10 \mathrm{~h} \mathrm{PW}$ ), the leading edges of epidermis are more migrated toward the wound center, and the p-MLC- and P-cadherin-positive areas are narrowed in the vicinity of the wound, while $\mathrm{K6}$ begin to be weakly expressed at the wound margin. The epidermal wound closure is completed around $12 \mathrm{~h} \mathrm{PW}$. Subsequently (12, $18 \mathrm{~h} \mathrm{PW),}$ the extraordinary (probably wound-specific) patterns of p-MLC and the P-cadherin expression fade out, whereas K6 becomes strongly expressed far from the wound areas. $e$, epidermis; $d$, dermis; $w$, wound; $b$, stratum basale; $s$, stratum spinosum; $g$, stratum granulosum; $c$, stratum corneum; arrows, the direction of locomotion; green lines, the expression of p-MLC; red frames, the expression of P-cadherin; and the red cells, $\mathrm{K} 6$ positive cells.

wound closure (Figures 3 and 8). Although the resolution of our optical system was insufficient definitive evidence, it is likely that, in our experimental system, p-MLC should be localized at the posterior lateral side of the individual cells for the contraction of their rear halves to facilitate the migration (Figure 3(M)), as inferred from the preceding researches on the cell motility of single cells [20-23]. The fact of the temporally and spatially specific emergence of p-MLC suggests that the cell migration driven by actomyosin system is involved in the wound closure of the shallow incisional wounds, leading us to speculate that, first, the epidermal cells at the basal and the spinous layers in the $10-20$ cells range from the wound edges are specified into "migration participants" for wound covering through some mechanism which is unclarified yet but undoubtedly involves the activation of actomyosin; second, these cells begin migrating, keeping the epithelial sheet architecture; and third, the migratory participant cells, in turn from the cells distant from the wound site, lose the motility a little before the completion of wound closure, and recover the normal state.

In addition, when the incisional wounds had been made on the adult skin, p-MLC was present at the epidermis migrating on the wound bed mechanically enlarged by the tension of the surrounding skin (Figure 5). This fact means that the actomyosin system is activated not only in the present shallow incisional wounds but also in the adult wounds including the epidermal crawling process.

Next, we explored the influences of Rho small GTPases inhibition on the epidermal wound closure in the present system. In the case of local administration of ROCK inhibitor Y27632, the wound closure was delayed (Figure 6), because of the hindrance of the migration of the epidermis from the wound edges via inactivation of phosphorylation of myosin light chain probably at the posterior end of cells. The application of Rac 1 inhibitor NSC23766 also resulted in a delay of wound closure, since the cells presumably failed to form lamellipodia at the anterior side, or lose the cell polarity, although it might be possible that phosphorylation of myosin light chain was indirectly enhanced, as Rac 1, known as an antagonist of Rho A, was inhibited by NSC23766 (Figure 7). Our data are consistent with the findings from the previous studies employing inhibition of Rho A or Rac 1 in vitro [24,25].

We observed that the wound closure was finished by $18 \mathrm{~h} \mathrm{PW}$ in both Y27632 and NSC23766 treated wounds (Figures 6(H) and 7(H)). Such an incompleteness of inhibition is likely to be due to the instability and/or the reversibility of the modes of action of these inhibitors [25-27].

The results from a series of inhibition experiments confirmed the above-stated view that both Rho A and Rac 1 are essential for the epidermal migration; concretely speaking, the wound closure is accomplished by the epidermal migration mediated by the Rho GTPasesdependent extension and contraction of epidermal cell bodies.

\subsection{Wound Closure May Be Executed by the Only Migration of Epidermis, Independently of Dermis}

The fact that small blister-like gaps were occasionally 
left in the dermis beneath the epidermis just after completion of wound closure, i.e., fusion of the leading edges (Figure 1) implies that the "epidermal" wound closure is apt to precede the "dermal" closure, and simultaneously supports the important possibility that, at least when the scale of the open wounds is small enough, epidermal cells can execute the wound closure by themselves without collaborative contribution of the underlying dermis. In other words, epidermal cells may be intrinsically capable of covering the open wounds, only cooperating their two intrinsic functions, first, maintenance of the cell-to-cell contact mediated by cadherin molecules (as will be further discussed again soon later) and, second, modulation of cell-shape and mobility driven by cytoskeletal molecules and the related factors such as Rho small GTPases.

\subsection{The Changes in the Expression Patterns of Keratin and Cadherin Are Correlated with the Wound Closure}

We have no direct evidence for contribution of K6 and $\mathrm{P}$-cadherin to the wound closure of the shallow incisions at present. However, some contributions of K6 and Pcadherin can be inferred from the fact that, roughly speaking, the occurrence of changes in expression or the emergences of both K6 and P-cadherin (Figure 2) during the wound closure were spatially coincident with that of p-MLC.

The expression sites of P-cadherin were expanded at 6 $\mathrm{h}$ PW, and gradually restored the normal pattern around the completion of the wound closure (Figures 2(G)-(L) and $\mathbf{8}(\mathbf{B})$ ). Since the expression of P-cadherin is restricted to the basal layer in normal epidermis, P-cadherin is considered as a marker of undifferentiated or progenitor cells [28-30]. Recent studies indicated that the overexpression of P-cadherin in breast cancer cells that were otherwise expressing E-cadherin induced an increased motility of the cells [31,32]. This reasoning may match the present multilayered epidermal system. Namely, the de novo expression of P-cadherin in the multilayered epidermis around the wound edges may contribute to the inward migration of the epidermal sheets by maintaining the undifferentiated state and the motility of the recruit cells to migration. However, we can deduce that the balanced regulation of the two apparently antagonistic activities, i.e., cell motility and maintenance of the epithelial sheet architecture, is rather important mission for the individual epidermal cells in the epidermal wound closure. In fact, the staining patterns of E-cadherin remained unchanged during the whole process of wound closure (Figures 2(A)-(F)), although the expression of P-cadherin is changed. Anyway, adherence junctions (AJ) via classical cadherins should be moderately maintained for the sheet-migration of epidermis.
K6 began to express weakly at the wound margins from $8 \mathrm{~h}$ PW onward when the epidermal sheets migrate, and its strong staining expanded up to the sites far from the wounds from $12 \mathrm{~h}$ PW when wound closure was finished (Figures 2(M)-(R), 8(B)). A recent study claimed that K6 suppresses the excessive cell migration, by binding to Src, which is involved in the migration of epidermal keratinocytes at wounds and tumors [33]. Hence we think that the strong staining of K6 observed around the wounds is indicative of the force to suppress the migration of epidermis after wound closure.

\subsection{The Patterns of Wound Closure of Shallow Incision Are Different between Neonates and Adults}

In adults, the shallow incisional wounds were not finished wound closure at $18 \mathrm{~h} \mathrm{PW}$ yet (Figure 5). This may be due to the extension of the wounds by tension of well-developed collagen fibers in dermis. In other words, it is thought that neonatal skin is beneficial for the analysis of the pure movement of epidermis in wound closure, since neonatal skin is less physically influenced by dermis than adult skin. The results obtained in this study are generally consistent with the previous findings that the patterns of epithelial wound healing change with developmental stages, and that the wound closure in neonates and fetus is faster than that in adults [4,34-38].

The shallow incisional wound model we developed in this study is an advantageous in vivo system allowing the analyses focusing the potential abilities of epidermal cells, by minimizing the influences of dermis. Using this model, we could provide morphological evidence that Rho small GTPases-mediated actomyosin is essential for the epidermal wound closure in rat neonates.

\section{Acknowledgements}

We thank the members of our Morphogenesis Laboratories for their support and helpful discussions in weekly seminars for furthering this study.

\section{REFERENCES}

[1] M. I. Koster and D. R. Roop, "Mechanisms Regulating Epithelial Stratification," Annual Review of Cell and Developmental Biology, Vol. 23, 2007, pp. 93-113. http://dx.doi.org/10.1146/annurev.cellbio.23.090506.1233 $\underline{57}$

[2] E. Proksch, J. M. Brandner and J. M. Jensen, "The Skin: an Indispensable Barrier," Experimental Dermatology, Vol. 17, No. 12, 2008, pp. 1063-1072. http://dx.doi.org/10.1111/j.1600-0625.2008.00786.x

[3] P. Martin and S. M. Parkhurst, "Parallels between Tissue Repair and Embryo Morphogenesis," Development, Vol. 
131, No. 13, 2004, pp. 3021-3034.

http://dx.doi.org/10.1242/dev.01253

[4] M. Koizumi, T. Matsuzaki and S. Ihara, "The Subsets of Keratinocytes Responsible for Covering Open Wounds in Neonatal Rat Skin," Cell and Tissue Research, Vol. 315, No. 2, 2004, pp. 187-195.

http://dx.doi.org/10.1007/s00441-003-0823-0

[5] M. Koizumi, T. Matsuzaki and S. Ihara, "Expression of P-Cadherin Distinct from That of E-Cadherin in Re-Epithelialization in Neonatal Rat Skin," Development, Growth \& Differentiation, Vol. 47, No. 2, 2005, pp. 75-85. http://dx.doi.org/10.1111/j.1440-169x.2004.00784.x

[6] M. Arai, T. Matsuzaki and S. Ihara, "Wound Closure on the Neonatal Rat Skin I. The Modulation of the Thickness of Epidermis at the Closing Incisional Wounds," CellBio, in Press.

[7] J. Brock, K. Midwinter, J. Lewis and P. Martin, "Healing of Incisional Wounds in the Embryonic Chick Wing Bud: Characterization of the Actin Purse-String and Demonstration of a Requirement for Rho Activation," The Journal of Cell Biology, Vol. 135, No. 4, 1996, pp. 10971107. http://dx.doi.org/10.1083/jcb.135.4.1097

[8] L. Van Aelst and M. Symons, "Role of Rho Family GTPases in Epithelial Morphogenesis," Genes \& Development, Vol. 16, No. 9, 2002, pp. 1032-1054. http://dx.doi.org/10.1101/gad.978802

[9] M. Fukata, M. Nakagawa and K. Kaibuchi, "Roles of Rho-Family GTPases in Cell Polarisation and Directional Migration," Current Opinion in Cell Biology, Vol. 15, No. 5, 2003, pp. 590-597. http://dx.doi.org/10.1016/S0955-0674(03)00097-8

[10] B. K. Chauhan, M. Lou, Y. Zheng and R. A. Lang, "Balanced Rac1 and RhoA Activities Regulate Cell Shape and Drive Invagination Morphogenesis in Epithelia," Proceedings of the National Academy of Science of the United States of America, Vol. 108, No. 45, 2011, pp. 1828918294. http://dx.doi.org/10.1073/pnas.1108993108

[11] E. E. Sander, J. P. ten Klooster, S. van Delft, R. A. van der Kammen and J. G. Collard, "Rac Downregulates Rho Activity: Reciprocal Balance between Both GTPases Determines Cellular Morphology and Migratory Behavior," The Journal Cell Biology, Vol. 147, No. 5, 1999, pp. 1009-1022. http://dx.doi.org/10.1083/jcb.147.5.1009

[12] A. S. Nimnual, L. J. Taylor and D. Bar-Sagi, "RedoxDependent Downregulation of Rho by Rac," Nature Cell Biology, Vol. 5, No. 3, 2003, pp. 236-241. http://dx.doi.org/10.1038/ncb938

[13] K. Burridge and K. Wennerberg, "Rho and Rac Take Center Stage," Cell, Vol. 116, No. 2, 2004, pp. 167-179. http://dx.doi.org/10.1016/S0092-8674(04)00003-0

[14] S. H. Baek, Y. C. Kwon, H. Lee and K. M. Choe, "RhoFamily Small GTPases Are Required for Cell Polarization and Directional Sensing in Drosophila Wound Healing," Biochemical and Biophysical Research Communications, Vol. 394, No. 3, 2010, pp. 488-492. http://dx.doi.org/10.1016/j.bbrc.2010.02.124

[15] K. H. Hyun, M. Nakai, K. Kawamura and M. Mori, "Histochemical Studies of Lectin Binding Patterns in Keratinized Lesions, Including Malignancy," Virchows
Archive. A, Pathological Anatomy and Histopathology, Vol. 402, No. 4, 1984, pp. 337-351.

http://dx.doi.org/10.1007/BF00734632

[16] G. Schaumburg-Lever, "Ultrastructural Localization of Lectin-Binding Sites in Normal Skin," The Journal of Investigative Dermatology, Vol. 94, No. 4, 1990, pp. 465470. http://dx.doi.org/10.1111/1523-1747.ep12874590

[17] D. L. Becker, I. McGonnell, H. P. Makarenkova, K. Patel, C. Tickle, J. Lorimer and C. R. Green, "Roles for Alpha 1 Connexin in Morphogenesis of Chick Embryos Revealed Using a Novel Antisense Approach," Developmental Genetics, Vol. 24, No. 1-2, 1999, pp. 33-42. http://dx.doi.org/10.1002/(SICI)1520-6408(1999)24:1/2< 33::AID-DVG5>3.0.CO;2-F

[18] R. Mori, K. T. Power, C. M. Wang, P. Martin and D. L. Becker, "Acute Downregulation of Connexin43 at Wound Sites Leads to a Reduced Inflammatory Response, Enhanced Keratinocyte Proliferation and Wound Fibroblast Migration," Journal of Cell Science, Vol. 119, No. 6, 2006, pp. 5193-5203. http://dx.doi.org/10.1242/jcs.03320

[19] S. Pellegrin and H. Mellor, “Actin Stress Fibres,” Journal of Cell Science, Vol. 120, No. Pt 20, 2007, pp. 34913499.

[20] J. Kolega, "Asymmetric Distribution of Myosin IIB in Migrating Endothelial Cells Is Regulated by a Rho-Dependent Kinase and Contributes to Tail Retraction," Molecular Biology of the Cell, Vol. 14, No. 12, 2003, pp. 4745-4757. http://dx.doi.org/10.1091/mbc.E03-04-0205

[21] A. J. Ridley, M. A. Schwartz, K. Burridge, R. A. Firtel, M. H. Ginsberg, G. Borisy, J. T. Parsons and A. R. Horwitz, "Cell Migration: Integrating Signals from Front to Back," Science, Vol. 302, No. 5651, 2003, pp. 17041709. http://dx.doi.org/10.1126/science. 1092053

[22] K. S. Uchida, T. Kitanishi-Yumura and S. Yumura, "Myosin II Contributes to the Posterior Contraction and the Anterior Extension during the Retraction Phase in Migrating Dictyostelium Cells," Journal of Cell Science, Vol. 116, No. Pt 1, 2003, pp. 51-60.

[23] A. Lee and J. E. Treisman, "Excessive Myosin Activity in Mbs Mutants Causes Photoreceptor Movement Out of the Drosophila Eye Disc Epithelium," Molecular Biology of the Cell, Vol. 15, No. 11, 2004, pp. 3285-3295. http://dx.doi.org/10.1091/mbc.E04-01-0057

[24] R. A. Worthylake and K. Brridge, "RhoA and ROCK Promote Migration by Limiting Membrane Protrusions," The Journal of Biological Chemistry, Vol. 278, No. 15, 2003, pp. $13578-13584$. http://dx.doi.org/10.1074/jbc.M211584200

[25] Y. Gao, J. B. Dickerson, F. Guo, J. Zheng and Y. Zheng, "Rational Design and Characterization of a Rac GTPaseSpecific Small Molecule Inhibitor," Proceedings of the National Academy of Science of the United States of America, Vol. 101, No. 20, 2004, pp. 7618-7623. http://dx.doi.org/10.1073/pnas.0307512101

[26] P. V. Rao, P. F. Deng, J. Kumar and D. L. Epstein, "Modulation of Aqueous Humor Outflow Facility by the Rho Kinase-Specific Inhibitor Y-27632," Investigative Ophthalmology \& Visual Science, Vol. 42, No. 5, 2001, 
pp. $1029-1037$.

[27] B. H. Chen, J. T. Tzen, A. R. Bresnick and H. C. Chen, "Roles of Rho-Associated Kinase and Myosin Light Chain Kinase in Morphological and Migratory Defects of Focal Adhesion Kinase-Null Cells," The Journal of Biological Chemistry, Vol. 277, No. 3, 2002, pp. 3385733863. http://dx.doi.org/10.1074/jbc.M204429200

[28] Y. Shimoyama, S. Hirohashi, S. Hirano, M. Noguchi, Y. Shimosato, M. Takeichi and O. Abe, "Cadherin CellAdhesion Molecules in Human Epithelial Tissues and Carcinomas," Cancer Research, Vol. 49, No. 8, 1989, pp. 2128-2133.

[29] J. Paredes, C. Stove, V. Stove, F. Milanezi, V. Van Marck, L. Derycke, M. Mareel, M. Bracke and F. Schmitt, "P-Cadherin Is Up-Regulated by the Antiestrogen ICI 182,780 and Promotes Invasion of Human Breast Cancer Cells," Cancer Research, Vol. 64, No. 22, 2004, pp. 8309-8317. http://dx.doi.org/10.1158/0008-5472.CAN-04-0795

[30] K. Raymond, M. A. Deugnier, M. M. Faraldo and M. A. Glukhova, "Adhesion within the Stem Cell Niches," Current Opinion in Cell Biology, Vol. 21, No. 5, 2009, pp. 623-629. http://dx.doi.org/10.1016/i.ceb.2009.05.004

[31] A. S. Ribeiro, A. Albergaria, B. Sousa, A. L. Correia, M. Bracke, R. Seruca, F. C. Schmitt and J. Paredes, "Extracellular Cleavage and Shedding of P-Cadherin: A Mechanism Underlying the Invasive Behaviour of Breast Cancer Cells," Oncogene, Vol. 29, No. 3, 2010, pp. 392402. http://dx.doi.org/10.1038/onc.2009.338

[32] A. Albergaria, A. S. Ribeiro, A. F. Vieira, B. Sousa, A. R. Nobre, R. Seruca, F. Schmitt and J. Paredes, "P-Cadherin
Role in Normal Breast Development and Cancer," The International Journal of Developmental Biology, Vol. 55, No. 7-9, 2011, pp. 811-822. http://dx.doi.org/10.1387/ijdb.113382aa

[33] J. D. Rotty and P. A. Coulombe, "A Wound-Induced Keratin Inhibits Src Activity during Keratinocyte Migration and Tissue Repair," The Journal Cell Biology, Vol. 197, No. 3, 2012, pp. 381-389. http://dx.doi.org/10.1083/jcb.201107078

[34] J. D. Burrington, "Wound Healing in the Fetal Lamb," Journal of Pediatric Surgery, Vol. 6, No. 5, 1971, pp. 523528. http://dx.doi.org/10.1016/0022-3468(71)90373-3

[35] J. B. Dixon, "Inflammation in the Foetal and Neonatal Rat: the Local Reactions to Skin Burns," The Journal of Pathology and Bacteriology, Vol. 80, 1960, pp. 73-82. http://dx.doi.org/10.1002/path.1700800109

[36] S. Ihara, Y. Motobayashi, E. Nagao and A. Kistler, "Ontogenetic Transition of Wound Healing Pattern in Rat Skin Occurring at the Fetal Stage," Development, Vol. 110, No. 3, 1990, pp. 671-680.

[37] M. T. Longaker, D. J. Whitby, N. S. Adzick, T. M. Crombleholme, J. C. Langer, B. W. Duncan, S. M. Bradley, R. Stern, M. W. Ferguson and M. R. Harrison, "Studies in Fetal Wound Healing, VI. Second and Early Third Trimester Fetal Wounds Demonstrate Rapid Collagen Deposition without Scar Formation," Journal of Pediatric Surgery, Vol. 25, No. 1, 1990, pp. 63-68, Discussion 8-9.

[38] P. Martin, "Mechanisms of Wound Healing in the Embryo and Fetus," Current Topics in Developmental Biology, Vol. 32, 1996, pp. 175-203. 